\title{
1RXS J173021.5-055933: a cataclysmic variable with a fast-spinning magnetic white dwarf ${ }^{\star}$
}

\author{
D. de Martino ${ }^{1}$, G. Matt ${ }^{2}$, K. Mukai ${ }^{3}$, J.-M. Bonnet-Bidaud ${ }^{4}$, M. Falanga ${ }^{4}$, B. T. Gänsicke ${ }^{5}$, F. Haberl ${ }^{6}$, T. R. Marsh ${ }^{5}$, \\ M. Mouchet ${ }^{7}$, S. P. Littlefair ${ }^{8}$, and V. Dhillon ${ }^{8}$
}

1 INAF - Osservatorio Astronomico di Capodimonte, via Moiariello 16, 80131 Napoli, Italy e-mail: demartino@na.astro.it

2 Dipartimento di Fisica, Universita' degli Studi Roma Tre, via della Vasca Navale 84, 00146 Roma, Italy e-mail: matt@fis.uniroma3.it

${ }^{3}$ CRESST and X-ray Astrophysics Laboratory NASA/GSFC, Greenbelt, MD 20771 and Department of Physics, University of Maryland, Baltimore County, 1000 Hilltop Circle, Baltimore, MD 21250, USA e-mail: mukai@milkyway.gsfc.nasa.gov

4 Service d'Astrophysique, DSM/DAPNIA/SAp, CE Saclay, 91191 Gif-sur-Yvette Cedex, France e-mail: [bonnetbidaud;mfalanga]@cea.fr

5 Department of Physics, University of Warwick, Coventry CV4 7AL, UK e-mail: [boris.gaensicke;Tom.Marsh]@warwick.ac.uk

${ }^{6}$ Max-Planck-Institut für Extraterrestrische Physik, Giessenbachstraße, Postfach 1312, 85741 Garching, Germany e-mail: fwh@mpe.mpg.de

7 Laboratory APC, University Denis Diderot, 10 Rue Alice Domon et Leonie Duquet, 75013 and LUTH, Observatoire de Paris, 92190 Meudon, France e-mail: martine.mouchet@obspm.fr

8 Department of Physics and Astronomy, University of Sheffield, Sheffield S3 7RH, UK e-mail: [s.littlefair;vik.dhillon]@shef.ac.uk

Received 27 July 2007 / Accepted 20 January 2008

\section{ABSTRACT}

\begin{abstract}
Aims. We present the first X-ray observations with the XMM-Newton and INTEGRAL satellites of the recently discovered cataclysmic variable 1RXS J173021.5-055933, together with simultaneous UV and coordinated optical photometry aiming at characterising its broad-band temporal and spectral properties and classifying this system as a magnetic one.

Methods. We performed a timing analysis of the X-ray, UV, and optical light curves to identify and to study the energy dependence of the fast $128 \mathrm{~s}$ pulsation over a wide energy range. X-ray spectral analysis in the broad $0.2-100 \mathrm{keV}$ X-ray range was performed to characterise the peculiar emission properties of this source.

Results. We find that the X-ray light curve is dominated by the spin period of the accreting white dwarf in contrast to the far-UV range, which turns out to be unmodulated at a $3 \sigma$ level. Near-UV and optical pulses are instead detected at twice the spin frequency. We identify the contributions from two accreting poles that imply a moderately inclined dipole field allowing, one pole to dominate at energies at least up to $10 \mathrm{keV}$, and a secondary that instead is negligible above $5 \mathrm{keV}$. X-ray spectral analysis reveals the presence of multiple emission components consisting of optically thin plasma with temperatures ranging from $0.17 \mathrm{keV}$ to $60 \mathrm{keV}$ and a hot blackbody at $\sim 90 \mathrm{eV}$. The spectrum is also strongly affected by peculiar absorption components consisting of two high-density $(\sim 3 \times$ $10^{21} \mathrm{~cm}^{-2}$ and $2 \times 10^{23} \mathrm{~cm}^{-2}$ ) intervening columns, plus a warm absorber. The last is detected from an OVII absorption edge at $0.74 \mathrm{keV}$, which suggests that photoionization of pre-shock material is also occurring in this system.

Conclusions. The observed properties indicate that the accretor in 1RXS J173021.5-055933 is a white dwarf with a likely weak magnetic field, thus confirming this cataclysmic variable as an intermediate polar (IP) with one of the most extreme spin-to-orbit period ratios. This system also joins the small group of IPs showing a soft X-ray reprocessed component, suggesting that this characteristics is not uncommon in these systems.
\end{abstract}

Key words. stars: binaries: close - stars: individual: 1RXS J173021.5-055933 - stars: novae, cataclysmic variables - white dwarfs

\section{Introduction}

Cataclysmic variables (CVs) are close binaries containing an accreting white dwarf (WD) from a late type Roche-lobe filling (main sequence or subgiant) secondary star (for a comprehensive review see Warner 1995). Among them, the magnetic systems (mCVs) nowdays constitute a rather conspicuous group ( $25 \%$

* Based on observations obtained with XMM-Newton and INTEGRAL, ESA science missions with instruments and contributions directly funded by ESA Member States and NASA. of all CVs). The current census of $\mathrm{mCVs}$, including confirmed ones and candidates, amounts to about 150 systems (Ritter \& Kolb (2006). They are further divided in two classes: the polars, containing a strongly magnetised (>10 MG) WD, whose rotation is synchronised with the orbital period and the intermediate polars (IPs), which contain accreting WDs that are rotating much faster than the orbital period. The presence of X-ray pulses at the WD rotation but the lack of detectable polarized circular radiation in the optical/near-infrared in most systems, led to believe that IPs possess weakly magnetised WDs ( $B \lesssim 10 \mathrm{MG})$ 
(see review by Patterson 1994). However this is still the subject of great debate (see below). As of today, the polars consist of $\sim 58 \%$ of the known mCVs. Because of their strong soft X-ray $(\sim 40 \mathrm{eV})$ emission component, the polars increased in number after the ROSAT era (Beuermann 1999). This was not the case for the IPs, which are dominated by a hard 10-20 keV optically thin emission. However in the last few years a large number ( $\sim 34)$ of IP candidates were discovered from ground-based observations (Warner \& Woudt 2004; Rodriguez-Gil et al. 2005; Gänsicke et al. 2005; Bonnet-Bidaud et al. 2006; Southworth et al. 2006; Southworth et al. 2007). Also, hard X-ray surveys such as those carried out by the INTEGRAL and SWIFT satellites are providing new and interesting results on CVs (Wheatley et al. 2006; Masetti et al. 2006a,b). In particular, from the current INTEGRAL catalogue (Bird et al. 2007) about 5\% of the detected sources are CVs, most of them are IPs (Barlow et al. 2006; Falanga et al. 2005). This has further stimulated optical and X-ray follow-ups to search for new IPs (e.g. Bonnet-Bidaud et al. 2007). The detection of high energy emission of IPs extending up to $\sim 90 \mathrm{keV}$ was already identified from BeppoSAX observations of a few bright systems (de Martino et al. 2001, 2004), which makes the new discoveries in the hard X-rays very promising to understand the role of this, formerly, small group of mCVs.

The link between polars and IPs, and hence the evolution of $\mathrm{mCVs}$, is still uncertain. Polars are typically found at short orbital periods, most of them below the so-called $2-3 \mathrm{~h}$ orbital period gap. IPs instead populate the orbital period distribution at longer periods with a handful of systems below the gap. The majority of IPs have rotational periods $\left(P_{\text {spin }}\right)$ about $7-10 \%$ the binary orbital periods $\left(P_{\text {orb }}\right)$. However, including the new optical candidates a wide range of degree of asynchronism, $P_{\text {spin }} / P_{\text {orb }}$ from $\sim 0.002$ to $\sim 0.7$, is encountered (Woudt \& Warner 2004; de Martino et al. 2006a). This might suggest that the former separation between IPs and polars could be only apparent and reinforces the debate on the hypothesis that IPs possess similar magnetic fields to those of the low-field polars, and will synchronise while evolving towards short orbital periods (Cumming 2002; Norton et al. 2004; Southworth et al. 2007). The confirmation of new optically discovered candidates is therefore essential in this respect. Differently from the optical range, heavily affected by X-ray reprocessing, a secure identification comes from the detection of X-ray pulses at the WD spin period, which arise from the post-shock region of the magnetically confined accretion flow onto the WD.

In the framework of a programme with XMM-Newton aiming at unambiguously confirming the IP nature of new optically identified systems, we here present the first X-ray study of 1RXS J173021.5-055933 (hereafter RXJ 1730). Identified as a magnetic CV by Gänsicke et al. (2005), its very fast (128 s) optical pulsations and very long $(15.4 \mathrm{~h})$ orbital period make it an extreme object in terms of degree of asynchronism. RX J1730 has the second fastest spinning WD preceeded only by AE Aqr with a spin period of $33 \mathrm{~s}$. Its orbital period is the second longest ever observed in $\mathrm{mCVs}$, being headed by GK Per with a period of 47.9 h. While GK Per and AE Aqr likely harbour K subgiant secondary stars (Morales-Rueda et al. 2002; Casares et al. 1996) the donor star in RX J1730 was suggested to be a G-type main sequence star, and differently from these two, the optical spectrum is dominated by the accretion flow rather than by the donor star (Gänsicke et al. 2005). The nature and evolutionary path of very long orbital period systems is still unknown though of great importance to understand the evolution of $\mathrm{mCVs}$ as a whole.
With the purpose of identifying unambiguously the rotational period of the WD primary and to study the emission and accretion properties of this system, the analysis presented here include a temporal and spectral analysis of the XMM-Newton observation. It was also detected in the hard X-rays during the IBIS/ISGRI galactic plane survey as IGR J17303-0601 =INTEGRAL1 56 (Bird et al. 2004) and formerly proposed to be a low mass X-ray binary (LMXB) by Masetti et al. (2004). Hence, we included in the spectral analysis also the archival data from the INTEGRAL satellite. We further complement the X-ray study with simultaneous far-UV photometry from the XMM-Newton Optical Monitor as well as coordinated quasi-simultaneous near-UV/optical photometry acquired at the William Herschel Telescope in La Palma.

\section{The observations}

\subsection{The XMM-Newton observation}

RX J1730 was observed with the XMM-Newton satellite (Jansen et al. 2001) on 2005 Aug. 29 (obsid:0302100201). The EPIC-PN (Strüder et al. 2001) and MOS (Turner et al. 2001) cameras were operated in large window mode with the medium filter for net exposure times of about $11.3 \mathrm{ks}$ and $13.3 \mathrm{ks}$ respectively. RX J1730 was also observed with the Reflection Grating Spectrographs (RGS1 and RGS2) (den Herder et al. 2001) in spectroscopy mode with an exposure time of $13.5 \mathrm{ks}$ and with the Optical Monitor (OM) instrument (Mason et al. 2001) using the wide $U V W 1$ filter covering the range 2450-3200 A (effective wavelength $2910 \AA$ ), in imaging fast mode for a total exposure time of $11.6 \mathrm{ks}$. A summary of the observations is reported in Table 1.

All data sets were reprocessed using the XMM-Newton SAS 6.5 package using the most updated calibration files. The EPIC light curves and spectra were extracted within the same SAS package from a circular region with a radius of $25^{\prime \prime}$ centred on the source. Background light curves and spectra were extracted from offset circular regions with same radii as for the target on the same CCD chip. Single and double pixel events with a zero quality flag were selected for the EPIC-PN data, while for EPIC-MOS cameras up to quadruple pixel events were used. The RGS pipeline was run using the SAS task rgsproc.

A long background flaring activity occurred $6.2 \mathrm{ks}$ after the start of the XMM-Newton observation. The average count rate during the flare is $0.13 \mathrm{cts} \mathrm{s}^{-1}$ and $0.02 \mathrm{cts} \mathrm{s}^{-1}$ in the EPIC-PN and MOS cameras respectively, reaching $0.6 \mathrm{cts} \mathrm{s}^{-1}(\mathrm{PN})$ and $0.2 \mathrm{cts} \mathrm{s}^{-1}$ (MOS) at flare maximum. Because the source was on average 26 and 10 times brighter than the background during the flare event does in both cameras respectively, the light curves are not greatly affected. On the other hand, since the source of flares are solar protons with unpredictable spectral behaviour, we conservatively limit the spectral analysis of the EPIC data to the first $6.2 \mathrm{ks}$ of observation. Also in the RGS instruments the count rate increases during flare up to $\sim 1 \mathrm{cts} \mathrm{s}^{-1}$. However the soft protons are not dispersed by the gratings and a comparison of the RGS spectra extracted including and without the flare periods, shows that the spectra are indeed not affected by this event. We therefore use for the RGS spectral analysis the whole observation. The EPIC-PN and MOS and the RGS1 and RGS2 first order spectra were rebinned to have a minimum of 20 counts in each bin. Ancillary response and redistribution matrix files were created using SAS tasks arfgen and rmfgen respectively.

OM background subtracted light curves were also produced using SAS from the reprocessed event files, extracting the source 
Table 1. Summary of the observations of RXJ1730.

\begin{tabular}{|c|c|c|c|c|}
\hline Instrument & Date & UT(start) & Duration (s) & Net Count Rate $\left(\right.$ cts s $\left.^{-1}\right)$ \\
\hline \multicolumn{5}{|l|}{ XMM-Newton } \\
\hline EPIC PN & 2005 Aug. 29 & 07:05 & 11271 & 3.73 \\
\hline EPIC MOS & & $06: 37$ & 13275 & 1.02 \\
\hline RGS & & $06: 36$ & 13507 & 0.12 \\
\hline \multirow[t]{5}{*}{ OM UVW1 } & & $06: 45$ & 2319 & 2.57 \\
\hline & & $07: 29$ & 2320 & \\
\hline & & $08: 13$ & 2320 & \\
\hline & & $08: 58$ & 2320 & \\
\hline & & 09:42 & 2319 & \\
\hline Instrument & Date & N. Science Window & $\operatorname{Duration}^{a}(\mathrm{~s})$ & Net Count Rate $\left(\right.$ cts s $\left.^{-1}\right)$ \\
\hline \multirow{9}{*}{ INTEGRAL/IBIS } & & & & 0.57 \\
\hline & 2003 Apr. $23-30$ & 75 & 135000 & \\
\hline & 2003 May 1 & 4 & 9182 & \\
\hline & 2003 Aug. 30 & 5 & 17757 & \\
\hline & 2003 Sep. 3 & 3 & 8637 & \\
\hline & 2003 Oct. $10-17$ & 95 & 172000 & \\
\hline & 2004 Feb. 15-25 & 28 & 61800 & \\
\hline & 2004 Mar. $11-20$ & 35 & 66700 & \\
\hline & 2004 Apr. 2 & 6 & 19875 & \\
\hline Instrument & Date & UT(start) & Duration (s) & $r^{\prime}$ (mag) \\
\hline \multicolumn{5}{|l|}{ William Herschel Telescope } \\
\hline \multirow[t]{6}{*}{ ULTRACAM $/ u^{\prime}, g^{\prime}, r^{\prime}$} & 2005 Aug. 27 & $20: 13$ & 2567 & 15.6 \\
\hline & 2005 Aug. 28 & $20: 30$ & 1925 & 15.6 \\
\hline & 2005 Aug. 29 & $20: 15$ & 3476 & 15.4 \\
\hline & 2005 Aug. 30 & $20: 19$ & 1255 & 15.3 \\
\hline & 2005 Aug. 31 & $20: 26$ & 992 & 15.3 \\
\hline & 2005 Sep. 1 & $20: 11$ & 1640 & 15.3 \\
\hline
\end{tabular}

${ }^{a}$ Total exposure time.

count rates from a circular region of $\sim 3^{\prime \prime}$ radius and the backgouund rates from an outer annulus with $\delta r \sim 2^{\prime \prime}$ centred on the source. Average net count rate in the $U V W 1$ filter is $2.57 \mathrm{cts} \mathrm{s}^{-1}$, corresponding to an instrumental magnitude of 16.3 mag. Using Vega magnitude to flux conversion, this corresponds to a flux of $1.2 \times 10^{-15} \mathrm{erg} \mathrm{cm}^{-2} \mathrm{~s}^{-1} \AA^{-1}$ in the 2450-3200 $\AA$ band. When compared with the extrapolation of the optical (4000-7000 $\AA$ ) spectra obtained by Gänsicke et al. (2005) at different epochs, the UV flux is higher than that observed in June 2003 but lower than that of April 2003. Heliocentric corrections were applied to the extracted light curves.

\subsection{The INTEGRAL data}

RX J1730 was detected as a hard X-ray source in the INTEGRAL (Winkler et al. 2003) IBIS/ISGRI (Ubertini et al. 2003; Lebrun et al. 2003) soft gamma-ray Galactic Plane Survey (Bird et al. 2004) with a count rate of $0.28 \pm 0.03 \mathrm{cts} \mathrm{s}^{-1}$ in the 20-40 keV range. As it will be shown in Sect. 3, the X-ray emission of RX J1730 is hard, with substantial emission above $10 \mathrm{keV}$. Hence, to extend the study to higher energies we analysed all public IBIS/ISGRI coded mask data in the range 20-100 keV. Several observing windows were included spanning from April 2003 to April 2004 (see Table 1). The data were extracted for all ISGRI pointings with a source position offset $\leq 9^{\circ}$ for a total effective exposure of $\sim 340 \mathrm{ks}$. Because of the weakness of the source and several short time windows, the data were only used to study the X-ray spectrum in the 20-100 keV range. The ISGRI spectrum was extracted using the standard INTEGRAL Offline Science Analysis (OSA) software version 5.1. Single pointings were deconvolved and analysed separately, and then combined in mosaic images. The source is clearly detected with a net count rate of $0.57 \mathrm{cts} \mathrm{s}^{-1}$ in the energy band $20-80 \mathrm{keV}$, at a significance level of $13.6 \sigma$. At energies above $100 \mathrm{keV} \mathrm{RX} \mathrm{J1730} \mathrm{was} \mathrm{not} \mathrm{detected} \mathrm{at} \mathrm{a} \mathrm{statistically}$ significant level in the total exposure time.

\subsection{The optical photometry}

RX J1730 was observed for six consecutive days at the $4.2 \mathrm{~m}$ William Herschel Telescope (WHT) at La Palma equipped with ULTRACAM (Dhillon et al. 2007), which provides imaging photometry at high temporal resolution in three different colours simultaneously. The observations were performed from Aug. 27 to Sept. 1, 2005 using the Sloan Digital Sky Survey (SDSS) $u^{\prime}$, $g^{\prime}, r^{\prime}$ filters with a temporal resolution of about $2 \mathrm{~s}$. The log of the observations is reported in Table 1, together with the average target magnitude level for each night. The seeing was variable in quality as well as transparency. The data were reduced using the ULTRACAM pipeline data-reduction system. Aperture photometry was performed on RX J1730 and several comparison stars. Differential photometry in the three bands was obtained by ratioing the background subtracted count rates of the target and best comparison star. Heliocentric correction was applied to the light curves in the three bands.

\section{Timing analysis}

\subsection{Search for periodicities}

To search for periodic variations in the X-ray band we extracted light curves in the $0.2-12 \mathrm{keV}$ range from the EPIC PN and MOS cameras with a time resolution of $2 \mathrm{~s}$. A Fourier analysis shows a strong peak at 675 day $^{-1}$ as well as at its first and third harmonic confirming the optical periodicity found by 


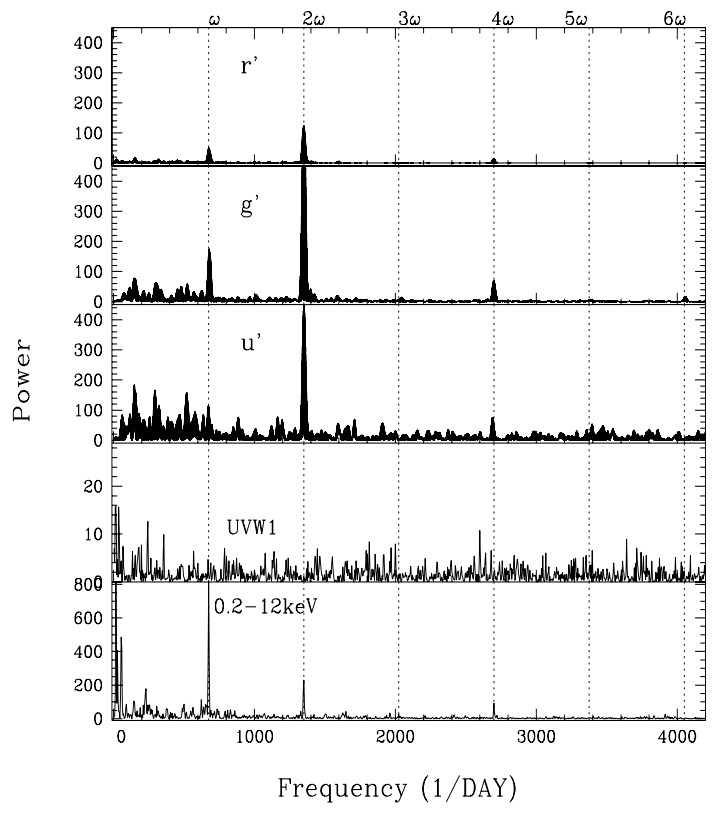

Fig. 1. Power spectra of RX J1730. From bottom to top: the EPIC-PN power spectra in $0.2-12 \mathrm{keV}$ range, in the $U V$ and $u^{\prime}, g^{\prime}, r^{\prime}$ bands, using light curves of Aug. 27, 28, and 29 (see text for details).

Gänsicke et al. (2005). This is shown in the bottom panel of Fig. 1 for the EPIC-PN light curve. The EPIC-MOS light curves are noisier but confirm this result. Hence the X-ray 128 s periodicity is confirmed to be the rotational period of the accreting WD. A sinusoidal fit to the EPIC-PN light curve with three sinusoids accounting for the fundamental, the first and third harmonics gives $P_{\omega}=128.02 \pm 0.02 \mathrm{~s}$ and the following ephemeris for the time of minimum of X-ray pulse: $\mathrm{HJD}_{\min }^{\mathrm{X}}=$ $2453611.86477(1))+0.0014817(2)$ E. We also performed a Fourier analysis on the extracted (10 s) light curves in selected energy bands as shown in Fig. 2. The fundamental dominates at all energies, while the first harmonic gets weaker at higher energies, indicating that the $\mathrm{X}$-ray pulse is more sinusoidal above $2 \mathrm{keV}$.

A search for periodic signals in the UVW1 light curve extracted with a temporal resolution of $2 \mathrm{~s}$ surprisingly did not show any variability above the $3 \sigma$ level (Fig. 1). This was also checked in each of the five UV data sets. We also inquired about any instrumental problem in the OM observation but no anomalies were found. Hence the lack of detectable variability in the $U V W 1$ filter should be regarded as real (but see also below).

The $u^{\prime}, g^{\prime}$, and $r^{\prime}$ light curves were also Fourier analysed to search for short periodic signals. The long term trend in each night was removed using a third order polynomial. In Fig. 3 we show the $r^{\prime}$ band power spectra for each night revealing that the $128 \mathrm{~s}$ modulation is present, but absent on Aug. 30. Furthermore while from Aug. 27 to Aug. 29 the first harmonic dominates over the fundamental and appears again on Aug. 31, on Sep. 1 only the fundamental is present. Strong and almost similar power at both fundamental and first harmonic was instead detected by Gänsicke et al. (2005). Changes from night to night or cycle to cycle of the amplitude of optical pulsations are not untypical of IPs (see Warner 1995, and references therein). The implications of the observed behaviour in the optical will be discussed in Sect. 5.

The XMM-Newton observation was carried out $10.5 \mathrm{~h}$ after the WHT/ULTRACAM run of Aug. 28 and $\sim 13 \mathrm{~h}$ before that of Aug. 29. Although further changes in the optical power

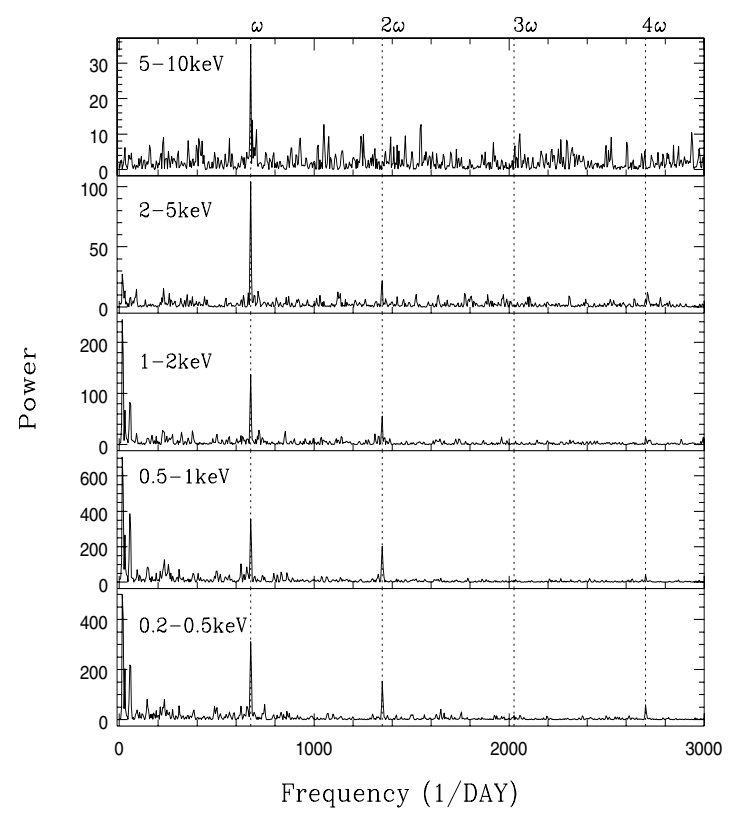

Fig. 2. EPIC-PN power spectra in selected energy ranges. From bottom to top: $0.2-0.5 \mathrm{keV}, 0.5-1 \mathrm{keV}, 1-2 \mathrm{keV}, 2-5 \mathrm{keV}$ and $5-10 \mathrm{keV}$. The spin $(\omega)$ and its harmonics are marked with vertical dotted lines. The fundamental dominates at all energies while the first harmonic is weak in the harder bands.

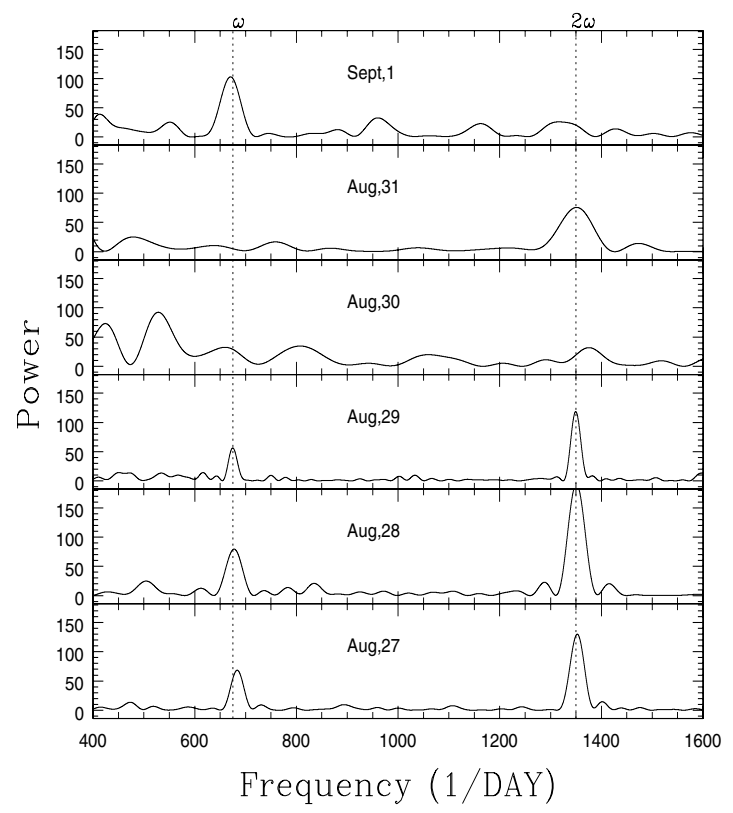

Fig. 3. Power spectra of the $r^{\prime}$ band light curves of RXJ1730 from Aug. 27 to Sep. 1, showing strong changes in the amplitude of the spin variability. Power at the fundamental frequency changes more than a factor of 2 from Aug. 31 to Sep. 1. Ordinates are arbitrary.

spectrum cannot be excluded, the dominance of the first harmonic from Aug. 27 to Aug. 29 allows us to assume that the optical behaviour is constant in these three days. We then assume that the X-ray power spectrum represents the behaviour of the X-ray source during that period, thus implying that the spin modulation is different in the two energy ranges. Figure 1 then reports a comparison between the X-ray and UV power spectra of Aug. 29 and those obtained from merging the optical $u^{\prime}$, $g^{\prime}, r^{\prime}$ band ones of Aug. 27-29. Interesting to note is the presence of the third harmonic not only in the X-rays but also in the optical and best visible in the $u^{\prime}$ and $g^{\prime}$ bands. In addition and 

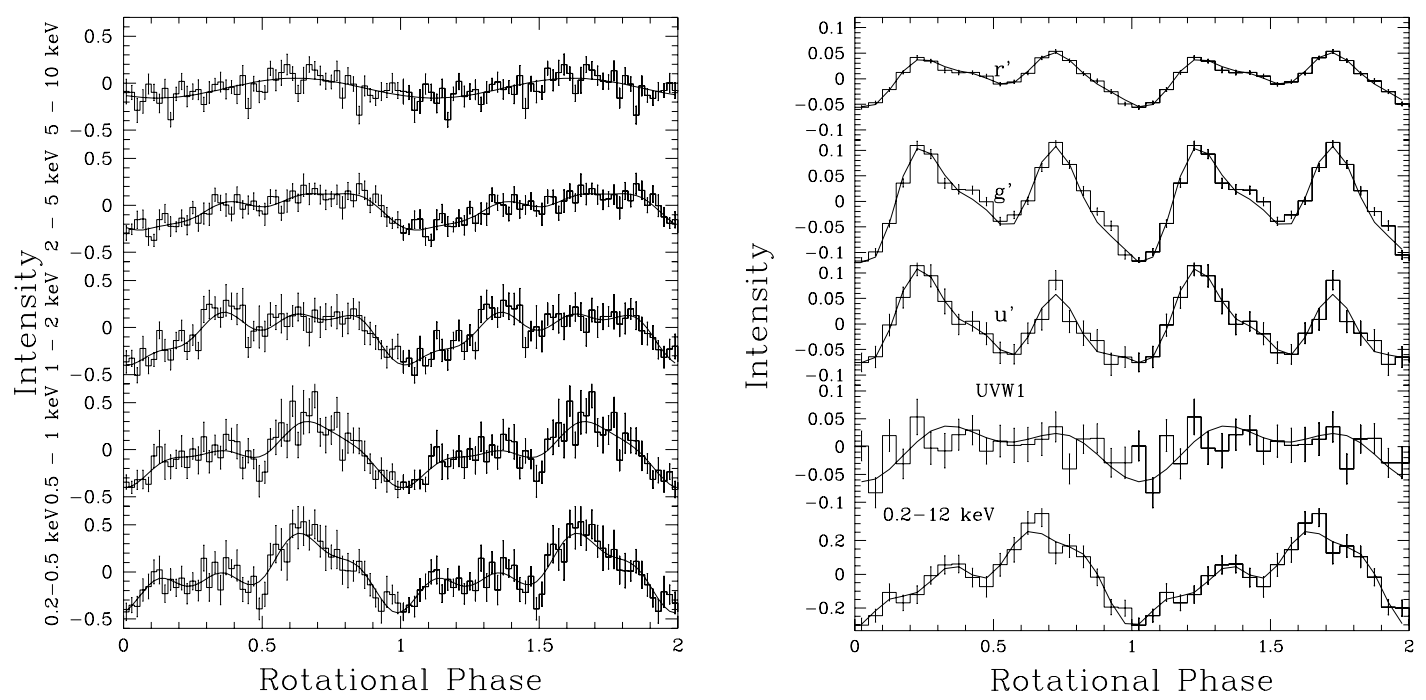

Fig. 4. Left: EPIC-PN folded light curves in selected energy bands at the 128 s period using the optical ephemeris quoted in the text. Right: a comparison of the X-ray, UV and optical, $u^{\prime}, g^{\prime}, r^{\prime}$ band spin light curves. Ordinates are modulation intensities. Sinusoidal fits consisting of three sinusoids at the fundamental frequency, the first and third harmonics are also reported, except for the hardest band $(5-10 \mathrm{keV})$ where only one sinusoid at the fundamental period is used, and the UVW1 band where two sines were used (see text).

contrary to the X-rays, power at the fifth harmonic is detected in the $g^{\prime}$ band, but not at the fourth. The fundamental frequency is below the $3 \sigma$ level in the $u^{\prime}$ band, but re-emerges in the $g^{\prime}$ and $r^{\prime}$ bands. Also, the power at the first harmonic is similar in the $u^{\prime}$ and $g^{\prime}$ bands while it has lower amplitude modulation in the red.

The detrended optical light curves of the first three nights were fitted with a composite function consisting of three sinusoids at the fundamental, first and third harmonics. For the $g^{\prime}$ band light curve, which provides the best fit, we obtain: $P_{\omega}=127.9999 \pm 0.0023 \mathrm{~s}$ and an ephemeris for the time of minimum of the optical spin pulse: $\mathrm{HJD}_{\min }^{\mathrm{opt}}=2453611.746278(4)+$ $0.00148148(3) \mathrm{E}$.

\subsection{The spin pulsation}

To compare the X-ray and optical spin pulsations we use the more accurate optical ephemeris for folding purposes. In Fig. 4 (left panel), we report the spin light curves in 50 phase bin points in different X-ray energy bands together with the best fit composite function with three sinusoids, at the fundamental, first and third harmonic frequencies (except for the 5-10 keV band, where only the fundamental is fitted). The amplitude of the modulation varies with energy being larger in the soft X-rays. In particular the spin pulse full amplitudes are: $48 \pm 2 \%$ in the $0.2-0.5 \mathrm{keV}$ band, $42 \pm 2 \%$ between $0.5-1 \mathrm{keV}, 24 \pm 1 \%$ in the $1-2 \mathrm{keV}$ and in the $2-5 \mathrm{keV}$ bands, and $10 \pm 1 \%$ in the $5-10 \mathrm{keV}$ range. The decrease in modulation amplitude with increasing energy is typically observed in IPs. Above $2 \mathrm{keV}$ the pulse has a broad maximum at $\phi_{\text {rot }} \sim 0.65$ where it softens, while it gets harder at minimum. This behaviour is consistent with photoelectric absorption in the accretion flow. The pulsation gets more structured below $2 \mathrm{keV}$ as indicated by the presence of higher harmonics in the power spectra.

The ratios of the amplitudes $A_{\omega} \sim 1.4 A_{2 \omega} \sim 2.4 A_{4 \omega}$ in the $0.2-1 \mathrm{keV}$ range and $A_{\omega} \sim 1.6 A_{2 \omega} \sim 4 A_{4 \omega}$ in the $1-5 \mathrm{keV}$ range, indicate that there is another emitting region offset by $180^{\circ}$, contributing $\sim 66 \%$ below $5 \mathrm{keV}$. The amplitude of the third harmonic is larger in the softest band suggesting either additional absorption or a contribution from a much softer region. Indeed and especially below $1 \mathrm{keV}$, a narrow dip at $\phi_{\text {rot }} \sim 0.5$ and a shallower one at $\phi_{\text {rot }} \sim 0.2$ can be recognised. The last is best seen in the soft $0.2-0.5 \mathrm{keV}$ range. Since as a hardening is detected at these phases, this suggests additional absorption effects at $\phi_{\text {rot }} \sim 0.2$.

For the comparison of the X-ray and optical spin pulses, we again used the detrended $u^{\prime}, g^{\prime}, r^{\prime}$ band light curves of Aug. 27-29. The folded light curves in the X-rays and optical bands are shown in the right panel of Fig. 4, together with the best sinusoidal fits that include three sinusoids with frequencies corresponding to the fundamental, the first and third harmonics. For the UV light curve we also show a two-sine fit, though the amplitudes are below the $3 \sigma$ level. Although the primary minimum occurs at about the same phases in both X-ray and optical bands, the pulse shape is different. The dip observed in the $\mathrm{X}$-rays at $\phi_{\text {rot }} \sim 0.5$ appears as a pronounced secondary minimum in the optical. The additional dip seen in the X-rays at $\phi_{\text {rot }} \sim 0.2$ is not observed in the optical where instead we observe a second maximum with a shoulder at $\phi_{\text {rot }} \sim 0.35$. The optical pulsed fraction is about the same in $u^{\prime}(13.5 \pm 0.7 \%)$ and $g^{\prime}$ bands (16.2 $\left.\pm 0.5 \%\right)$, while it is $7 \pm 0.1 \%$ in the $r^{\prime}$ band. This last is consistent with that observed by Gänsicke et al. (2005). On the other hand, the amplitude in the $U V W 1$ band is $7.1 \pm$ $2.6 \%$ and hence a hardly detectable modulation in this range. The differences of the optical spin pulse in the three bands are essentially due to the different amplitudes of first and third harmonics, being $A_{\omega} \sim 0.5 A_{2 \omega} \sim 1.8 A_{4 \omega}$ in the $u^{\prime}$ and $g^{\prime}$ bands, while in the $r^{\prime}$ band $A_{\omega} \sim 0.6 A_{2 \omega} \sim 2.4 A_{4 \omega}$. As a further comparison, taking at face values the amplitudes of the fundamental and first harmonic in the $U V W 1$ band, $A_{\omega} \sim 1.4 A_{2 \omega}$ are rather similar to what observed in the X-rays. This could suggest that the overall UV/optical pulse is not very blue and that only longwards $3000 \AA$, it is dominated by two equally contributing regions. We also note an asymmetry in the optical pulse profile at $\phi_{\text {rot }} \sim 0.35$ suggesting an additional contribution of $\sim 26 \%$ to the optical modulation. This will be discussed in Sect. 5 .

\section{The X-ray spectrum of RX J1730}

Identification of spectral components was performed on the XMM-Newton EPIC PN and the combined MOS averaged 

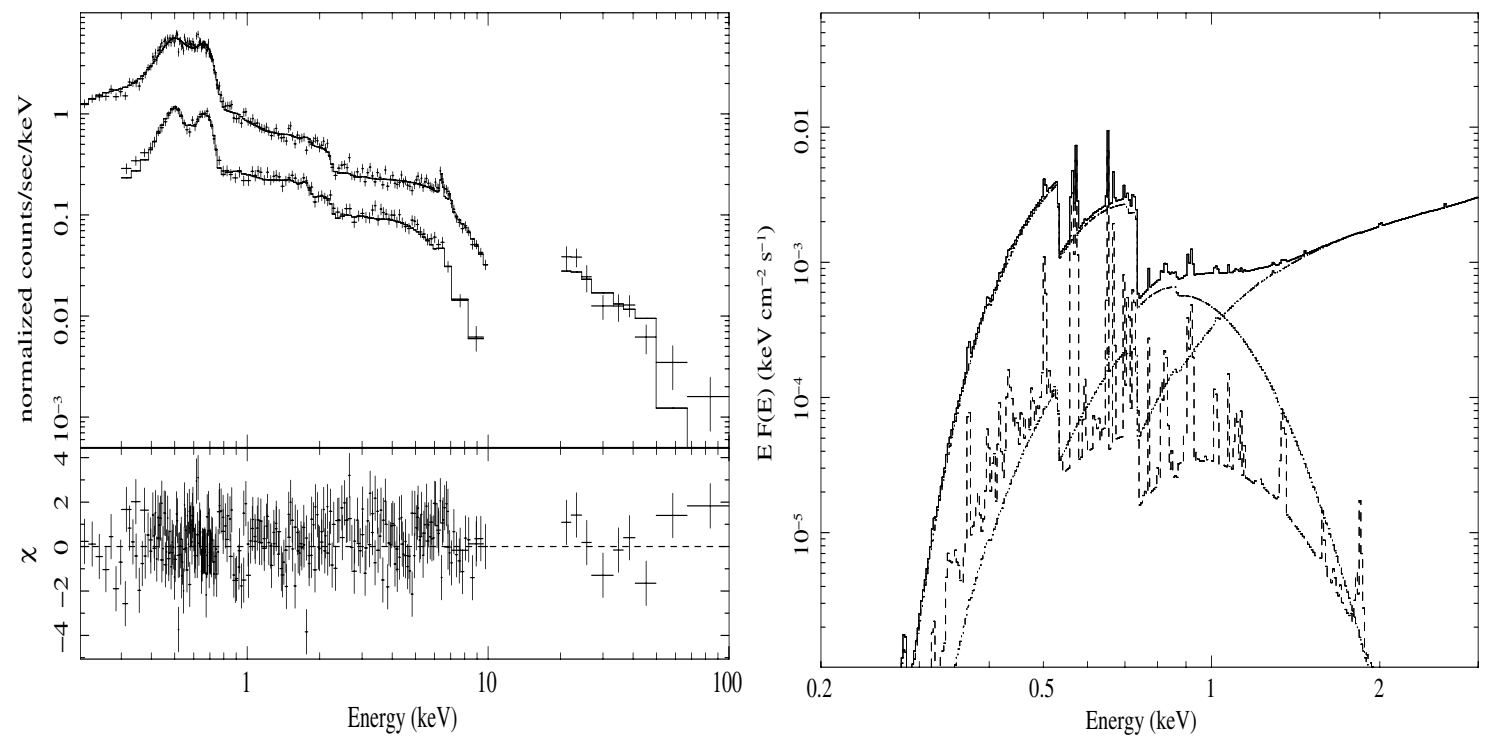

Fig. 5. Left: the EPIC PN (top) and combined MOS (bottom) and IBIS spectra fitted simultaneously with the multicomponent model B (see text). The lower panel reports the residuals between predicted and observed spectra. Right: the unfolded model including the contribution of various components is plotted up to $2 \mathrm{keV}$, showing the complexities in the soft portion of the spectrum.

Table 2. A: Spectral parameters as derived from fitting simultaneously the EPIC PN and MOS phase-averaged spectra for the best fit model discussed in the text. Quoted errors refer to $90 \%$ confidence level for the parameter of interest. B refers to the inclusion of IBIS/ISGRI spectrum.

\begin{tabular}{|c|c|c|c|c|c|c|c|c|c|c|c|}
\hline & $N_{\mathrm{H}}^{a}$ & $N_{\mathrm{H}}^{b}$ & $\operatorname{Cov}_{\mathrm{F}}^{c}$ & $\begin{array}{c}E_{\mathrm{c}} \\
(\mathrm{keV})\end{array}$ & $\tau_{\max }$ & $\begin{array}{l}k T_{\mathrm{BB}} \\
(\mathrm{eV}) \\
\end{array}$ & $A_{Z}^{d}$ & $\begin{array}{c}k T_{1} \\
(\mathrm{keV}) \\
\end{array}$ & $\begin{array}{c}k T_{2} \\
(\mathrm{keV})\end{array}$ & $\begin{array}{l}E W^{e} \\
(\mathrm{eV})\end{array}$ & $\chi^{2} /$ d.o.f. \\
\hline $\mathbf{A}$ & $3.57_{-0.25}^{+0.24}$ & $1.40_{-0.17}^{+0.32}$ & $0.56_{-0.02}^{+0.03}$ & $0.740 \pm 0.005$ & $1.82_{-0.16}^{+0.17}$ & $79.5_{-3.3}^{+3.4}$ & $0.33_{-0.19}^{+0.37}$ & $0.174_{-0.015}^{+0.006}$ & $>59.5$ & $114 \pm 29$ & $924 / 928$ \\
\hline B & $3.11_{-0.06}^{+0.03}$ & $2.03_{-0.30}^{+0.19}$ & $0.56 \pm 0.02$ & $0.736_{-0.006}^{+0.002}$ & $1.65_{-0.06}^{+0.07}$ & $91.4_{-0.8}^{+0.9}$ & $0.40_{-0.08}^{+0.067}$ & $0.168_{-0.003}^{+0.015}$ & $60 \pm 6$ & $107 \pm 30$ & $898 / 937$ \\
\hline
\end{tabular}

${ }^{a}$ Column density of total absorber in units of $10^{21} \mathrm{~cm}^{-2}$.

${ }^{b}$ Column density of the partial absorber in units of $10^{23} \mathrm{~cm}^{-2}$.

${ }^{c}$ Covering fraction of partial absorber.

${ }^{d}$ Metal abundance in units of the cosmic value (Anders \& Grevesse 1989) linked for the two thin plasma emissions.

${ }^{e}$ Equivalent width of gaussian centred at $6.4 \mathrm{keV}$.

spectra using the XSPEC package. We extracted the spectra between 0.3 and $10 \mathrm{keV}$, outside which the calibration accuracy of the MOS cameras is low. Also, because of the non simultaneity of the INTEGRAL observations, the INTEGRAL/ISGRI spectrum was included in the spectral analysis at a later stage (see Fig. 5 and Table 2).

The X-ray spectrum below $1 \mathrm{keV}$ is characterised by a broad feature between 0.4 and $0.7 \mathrm{keV}$ followed by a cut-off, whilst at high energies, it shows the well known iron complex including the fluorescent $6.4 \mathrm{keV}$ line and a hard tail. The soft portion of the spectrum is very atypical for IPs (e.g. Haberl \& Motch 1995; Mukai et al. 1995; Ramsay et al. 1998; Haberl et al. 2002; Evans \& Hellier 2004; de Martino et al. 2004, 2006a,b; Evans et al. 2006).

The EPIC spectra require two optically thin components as well as a blackbody emission absorbed by multiple components. Acceptable fits are achieved including two MEKAL emissions, a blackbody and three absorption components. The last consist of a total (WABS), a partial (PCFABS) absorbers, and an absorption EDGE, defined as $\mathrm{e}^{-\tau\left(E / E_{\mathrm{c}}\right)^{3}}$ for $E \geq E_{\mathrm{c}}$, where $\tau$ the optical depth at threshold energy $E_{\mathrm{c}}$ and 1 for $E \leq E_{\mathrm{c}}$. With model A we then define a composite spectrum: WABS *PCFABS *EDGE(MEKAL+BBODY + MEKAL+ GAUSSIAN), where the gaussian line accounts for the iron fluorescent line fixed at $6.4 \mathrm{keV}$. This model gives $\chi_{v}^{2}=0.995$ and in Table 2, A, we report the spectral parameters. The energy at which this edge is found $(0.74 \mathrm{keV})$ implies ovII K-shell absorption with an optical depth at this energy $\tau \sim 1.8$. No OVIII edge, expected at $0.87 \mathrm{keV}$, is observed. This absorption feature is indicative of the presence of a warm absorber along the line of sight. However, the description with an ionised absorber ABSORI (Done et al. 1992) does not provide an equally acceptable fit $\left(\chi_{v}^{2}=1.09\right)$, leaving the gas parameters unconstrained. The total absorber has an column density about twice that of the Galactic absorption in the direction of the source $\left(N_{\mathrm{H}}=1.7 \times 10^{21} \mathrm{~cm}^{-2}\right)$, indicating an intrinsic contribution of the source. The second intervening column has a density $N_{\mathrm{H}}=1.4 \times 10^{23} \mathrm{~cm}^{-2}$ and covers $\sim 56 \%$ of the X-ray source. Multiple absorption components are indeed observed in many X-ray spectra of IPs (Mukai et al. 1994). The optically thick component is rather hot $\left(k T_{\mathrm{BB}}=80 \mathrm{eV}\right)$ when compared with those observed in the polars and the soft X-ray IPs, but it is similar to that found in some hard IPs (Haberl et al. 2002; de Martino et al. 2004, 2006a). The inclusion of the blackbody 
under only the partial absorber provides a worse fit $\left(\chi_{v}^{2}=1.04\right)$. The two optically thin components are found at $k T_{1}=0.17 \mathrm{keV}$ and $k T_{2} \geq 60 \mathrm{keV}$ with sub-solar metal abundance $A_{Z}=0.33$. A multi-temperature plasma (CEMEKL) is not required by the data, providing a $\chi_{v}^{2}>2$ even when including the INTEGRAL/ISGRI data. This indicates that the power law temperature dependence of the emission measure is a poor description of the post-shock region. A similar result was also found in other systems (e.g. Evans \& Hellier 2004; de Martino et al. 2006b). However, the iron complex in the $6-7 \mathrm{keV}$ region was inspected against the presence of intermediate temperatures by adopting a hot MEKAL at $k T_{2}=60 \mathrm{keV}$ with $A_{Z}=0.33$ plus a Gaussian centred on the $6.4 \mathrm{keV}$ line and the absorption parameters fixed at the values of model A). The model predicts the hydrogenic $6.97 \mathrm{keV}$ iron line but, as expected, not the He-like iron line at $6.7 \mathrm{keV}$. Inclusion of a gaussian line at $6.7 \mathrm{keV}$ gives $E W=44 \pm 8 \mathrm{eV}$. This emission line can be accounted for by a $10 \mathrm{keV}$ plasma, suggesting that there are regions of the optically thin post-shock plasma at lower temperatures.

The inclusion of the INTEGRAL/ISGRI spectrum lowers the hot temperature MEKAL to $60 \mathrm{keV}$ (see Table 2, B). The uncertainty of the instrument flux calibration was taken into account by introducing a multiplicative constant into the spectral model and normalizing the fluxes to the PN. This constant was 0.92. Again the metal abundance results to be sub-solar $A_{Z}=$ $0.40_{-0.08}^{+0.06}$. We remark that it is relative to Anders \& Grevesse (1989) solar abundances that are higher than those adopted for the interstellar medium (e.g. Wilms et al. 2000; but also see Grevesse et al. 2007). The differences between the two are still within errors. The flux in the $0.2-10 \mathrm{keV}$ range is $1.68 \pm 0.02 \times$ $10^{-11} \mathrm{erg} \mathrm{cm}^{-2} \mathrm{~s}^{-1}$. This fit is reported in the left panel of Fig. 5 and in the right panel we show the unfolded model together with the different spectral components.

The high temperature found for the hot optically thin component and the relatively large width $(E W=110 \mathrm{eV})$ of the fluorescent $\mathrm{K}_{\alpha}$ iron line at $6.4 \mathrm{keV}$ could suggest illumination from cold material due to Compton reflection (Matt et al. 1991; Done et al. 1992). This component has been detected in many magnetic CVs (Matt et al. 2000; de Martino et al. 2001; Ramsay \& Cropper 2004) and alleviates the problem of high plasma temperatures. However, including this component in the fit, (Magdziarz \& Zdziarski 1995) no improvement is found $\left(\chi_{v}^{2}=0.98\right)$. We are then left with an uncomfortably high plasma temperature that might be regarded as representative of the immediate post-shock regions of the accretion flow.

The observed complexity in the $0.6 \mathrm{keV}$ region and hence the cool MEKAL can also be reproduced by a gaussian centred at $0.568 \mathrm{keV}(21.81 \AA$ ) implying that O VII He-like triplet line is present. We then analysed the RGS spectra applying the same model used for the EPIC data to both RGS1 and RGS2 spectra, but this predicts too much flux in the O VII line. Because of the low data quality, we left free to vary only the total absorber and MEKAL abundance, while keeping all other parameters fixed at the values reported in Table 2,B. The major difference was found in the abundance, which is lowered to $A_{Z}=0.21$. The RGS spectra are shown in Fig. 6 together with the expected positions of main emission lines. Worth noticing is the strong O VII edge at $\sim 17 \AA$, which is the main feature of the RGS spectrum and the lack of strong lines. The OVIII (at $19 \AA$ ) line might be present though reliable parameters are difficult to derive due to its weakness and broadness $\sim 30 \mathrm{eV}$. We also included the ABSORI component in place of the edge and find that this component is able to reproduce the observed spectrum ("bump") in the 15-20

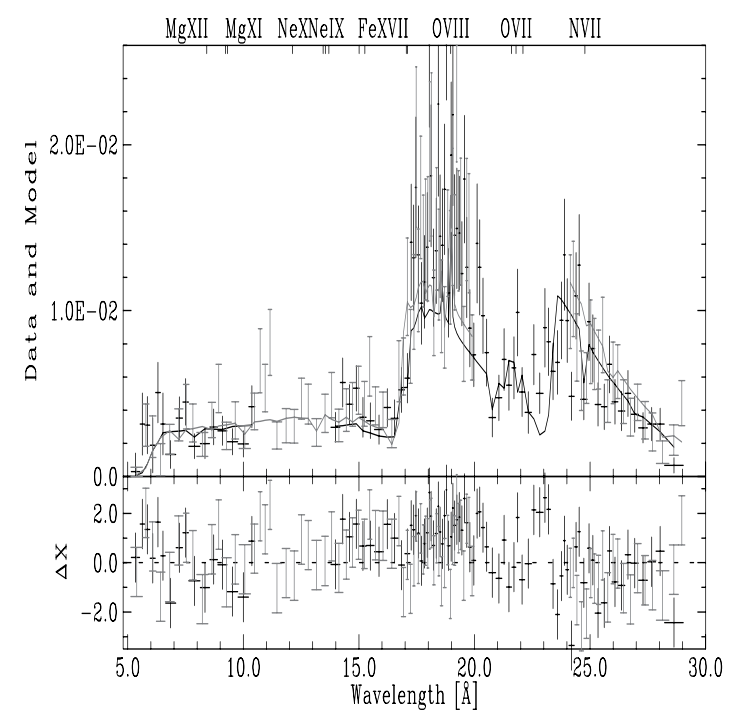

Fig. 6. The RGS1 (black) and RGS2 (gray) spectra fitted with model parameters derived from the combined EPIC and ISGRI spectral analysis, but with lower abundance $\left(A_{Z}=0.21\right)$ as discussed in the text. The positions of lines expected to be strong are also reported. The residuals between model and observed spectrum are shown in the lower panel.

region, though also in this case we are unable to derive constraints on ionization parameters. In summary the RGS spectra confirm the presence of a warm absorber in RX J1730.

\section{Discussion}

Our XMM-Newton observation of RX J1730 complemented with high energy INTEGRAL data and with optical WHT photometry revealed new properties of this intriguing $\mathrm{CV}$. The first detection of strong X-ray pulses at the previously identified optical $128 \mathrm{~s}$ period confirms that this is one of the most rapidly rotating WDs among IPs and that its X-ray emission is one of the most complex ever observed.

\subsection{The emission properties}

We detected atypical spectral properties in RX J1730. Our combined XMM-Newton and INTEGRAL analysis has shown that it is a very hard source with complex low energy properties. The high energy portion of the spectrum is dominated by the hottest post-shock regions of up to temperatures of $\sim 50-60 \mathrm{keV}$. These values, if taken as representative of the shock itself, would imply $M_{\mathrm{WD}}=0.89-1.02 M_{\odot}$ and hence a rather massive primary. The low energy part of the X-ray spectrum reveals the presence of absorbed soft components: a blackbody of similar temperature $(90 \mathrm{eV})$ as found in other hard X-ray IPs observed with XMM-Newton and BeppoSAX (e.g. Haberl et al. 2002; de Martino et al. 2004, 2006a), but much hotter than that detected in the "classical" soft IP systems (30-50 eV) (Haberl \& Motch 1995; de Martino et al. 2004, 2006b), and a relatively cool optically thin component at $0.17 \mathrm{keV}$. The last was also identified in the XMM-Newton spectra of the soft systems V405 Aur (Evans \& Hellier 2004), PQ Gem (Evans et al. 2006) and UUCol (de Martino et al. 2006b). This component is further confirmed by the detection of the OVII He-like emission line in the RGS spectrum. The absorption in RX J1730 is very complex, consisting of two high density intervening columns with 
$N_{\mathrm{H}} \sim 3 \times 10^{21} \mathrm{~cm}^{-2}$ and $N_{\mathrm{H}} \sim 2 \times 10^{23} \mathrm{~cm}^{-2}$, the last covering $\sim 56 \%$ the $\mathrm{X}$-ray source, as well as of a ionised absorber detected from the K-shell absorption edge of o VII. The last has a relatively high optical depth $(\tau \sim 1.7)$. Using the photoionization cross section of OVII (Verner et al. 1996), we derive a column density for $\mathrm{O}$ VII of $\sim 7 \times 10^{18} \mathrm{~cm}^{-2}$. Assuming that this represents all the oxygen atoms, the equivalent total hydrogen column, for a solar abundance of oxygen $8.51 \times 10^{-4}$ (Anders \& Grevesse 1989), is $8 \times 10^{21} \mathrm{~cm}^{-2}$. This value is similar to that obtained using the warm absorber model. The material has a relatively high ionization level and might be also partially responsible for the red-shifted o VII line due to photoionization. While absorption edges are often detected in AGN nuclei and QSOs (Piconcelli et al. 2004, 2005), the first unambiguous detection of a warm absorber in an IP was found in the Chandra spectrum of V1223 Sgr (Mukai et al. 2001), where a similar O VII edge was detected. A second detection of an absorption edge, but from ionised iron, was found in the IP V709 Cas (de Martino et al. 2001) from Rossi XTE observations. As proposed by Mukai et al. (2001), photoionization in the pre-shock flow might occur when the shock height is small compared to the diameter of the flow. It could be also occurring in RX J1730, as this system could be a weak magnetic field accretor (see below). Due to the lack of adequate temporal coverage it is not possible to further explore this hypothesis via phase-resolved spectroscopy, since the warm absorber feature should be deepest when viewing the post-shock region through the pre-shock flow.

The increasing evidence that IPs also possess a nonnegligible, but heavily absorbed, soft X-ray blackbody component is a challenging new result that BeppoSAX and $X M M$-Newton data recently brought into light. Whether IPs show this component because of geometric factors has been recently discussed by Evans \& Hellier (2007). The bolometric blackbody flux $F_{\mathrm{BB}}=1.31 \times 10^{-10} \mathrm{erg} \mathrm{cm}^{-2} \mathrm{~s}^{-1}$, when compared with that of the optically thin components $F_{\text {thin }}=1.5 \times$ $10^{-10} \mathrm{erg} \mathrm{cm}^{-2} \mathrm{~s}^{-1}$, yields a ratio of blackbody to plasma emission of $87 \%$. Comparing this ratio with those found in other IPs (Haberl et al. 2002; de Martino et al. 2004, 2006a,b), there appears to be a tendency for higher ratios for hotter blackbodies, reflecting the strong dependence on the temperature. These high temperatures could arise from small "cores" of the heated magnetic polar areas on the WD surface. In RX J1730 the blackbody bolometric luminosity $L_{\mathrm{BB}}=1.6 \times 10^{34} d_{1 \mathrm{kpc}}^{2} \mathrm{erg} \mathrm{s}^{-1}$ is very high. This gives an emitting area $a_{\mathrm{BB}}=2.3 \times 10^{14} d_{1 \mathrm{kpc}}^{2} \mathrm{~cm}^{2}$ and a fractional WD area $f \sim 3 \times 10^{-5} d_{1 \mathrm{kpc}}^{2}$. Though this parameter is subject to model uncertainties, it is by three orders of magnitude lower than those derived in the soft X-ray IPs showing cooler blackbodies such as PQ Gem (James et al. 2002). However, it is larger by one order of magnitude than that in NY Lup that shows a similar hot blackbody component (Haberl et al. 2002).

The emission measure of the optically thin plasma, EM $24 \times 10^{55} d_{1 \mathrm{kpc}}^{2} \mathrm{~cm}^{-3}$, also turns out to be large if the system is at $1 \mathrm{kpc}$. This might favour high densities as also suggested by the large column densities and high mass accretion rate (see below).

As far the distance is concerned, Gänsicke et al. (2005) estimate a donor star of type G0V-G6V contributing $15 \%$ of the total flux at $6800 \AA$ and a reddening $E_{B-V}=0.45$. This reddening is consistent with the hydrogen column density derived for the total absorber from the X-ray spectral analysis. They also derive an amplitude of $115 \mathrm{~km} \mathrm{~s}^{-1}$ from He II emission line radial velocity curve. Assuming that this represents the motion of the primary star in a $15.4 \mathrm{~h}$ orbit, we infer a mass function $f\left(M_{\mathrm{sec}}\right)=$ $0.101 M_{\odot}$. Using the WD mass estimate $0.89-1.02 M_{\odot}$, the secondary mass would then be in the range $M_{\mathrm{sec}}=0.62-0.92 M_{\odot}$ for inclination angles $i \sim 50-90^{\circ}$. Lower values of the inclination angle $\left(i<50^{\circ}\right)$ would imply a donor star more massive than the primary. Further constraints on the system geometry are derived below. The secondary radius, assuming a Roche-lobe filling star, would be in the range $\sim 1.2-1.4 R_{\odot}$ for a $15.4 \mathrm{~h}$ orbital period. Because of the dominant contribution of accretion to the optical flux, we use the infrared $K$ band measure from 2MASS survey reported in Gänsicke et al. (2005) to derive a lower limit to the distance of RX J1730. This because the secondary star is expected to dominate the IR flux, although it cannot be excluded that cyclotron emission, outer disc or circumbinary cool material (Taam \& Spruit 2001) could contribute to the $K$ band flux. Hence, adopting a G0-G6 type donor star with $R_{\mathrm{sec}}=1.0-1.4 R_{\odot}$, the reddening reported above, and using the $K$ band surface brightness, we derive $d \geq 1.0-1.6 \mathrm{kpc}$. Though it is the minimum distance, the assumed value of $8 \mathrm{kpc}$ by Masetti et al. (2004) would imply a bolometric luminosity much larger (by two orders of magnitude) than typical values of CVs in quiescence.

The total luminosity, including the soft and hard components results to be $L_{\mathrm{BOL}}=3.36 \times 10^{34} d_{1 \mathrm{kpc}}^{2} \mathrm{erg} \mathrm{s}^{-1}$. This translates into a mass accretion rate of $\sim 2.2-2.5 \times 10^{-9} d_{1 \mathrm{kpc}}^{2} M_{\odot} \mathrm{yr}^{-1}$ for $M_{\mathrm{WD}}=0.89-1.02 M_{\odot}$. While this is a high value if the source is indeed at a distance of $1 \mathrm{kpc}$, it is not unexpected for its long orbital period. To this regard we note that the average secular mass accretion rate for systems above the orbital period gap is $\sim 10^{-9} M_{\odot} \mathrm{yr}^{-1}$ (McDermott \& Taam 1989), as the result of magnetic braking of the secondary star that acts as angular momentum loss mechanism in these systems. As a comparison, the long period IP NY Lup $\left(P_{\text {orb }}=9.87 \mathrm{~h}\right)$ was found at a mass accretion rate of $\sim 8 \times 10^{-10} M_{\odot} \mathrm{yr}^{-1}$ (de Martino et al. 2006c). At a similar orbital period, the peculiar AE Aqr $\left(P_{\text {orb }}=9.88 \mathrm{~h}\right)$, is not in an accretion regime but in a propeller state with a secondary transferring mass at a rate of $\sim 6.4 \times 10^{-9} M_{\odot} \mathrm{yr}^{-1}$ (see also Venter \& Meintjes 2007). Since, RX J1730 shows all signatures of an accreting magnetic WD, the estimated rate is not too far from what expected from magnetic braking.

Furthermore, accretion from the stellar wind of the G-type donor star is not expected to be the main source of the observed X-ray luminosity. This because adopting a power law dependence of the mass loss rate on the surface magnetic field strength (Collier Cameron 2002) and a secondary star surface magnetic field of $115 \mathrm{G}$, derived from the donor rotational (orbital) period, a wind mass loss rate of $\sim 5 \times 10^{-11} M_{\odot} \mathrm{yr}^{-1}$ is estimated. Indication of a high accretion rate also comes from the large contribution of the accretion flow in the red band (Gänsicke et al. 2005) where, instead, long-period systems are less affected. It might also favour an extended accretion disc consistent with the wide orbit of the system. The condition for disc formation and truncation at the magnetospheric boundary is $R_{\text {mag }} \sim R_{\mathrm{co}}$, where $R_{\mathrm{co}}=\left(\mathrm{G} M_{\mathrm{WD}} P_{\omega}^{2} / 4 \pi^{2}\right)^{1 / 3} \sim 3.7 \times 10^{9} \mathrm{~cm}$ is the corotation radius at which the magnetic field rotates with the same Keplerian frequency of the WD and where $R_{\text {mag }}=5.5 \times$ $10^{8}\left(M_{\mathrm{WD}} / M_{\odot}\right)^{1 / 7} R_{9}^{-2 / 7} L_{33}^{-2 / 7} \mu_{30}^{4 / 7} \mathrm{~cm}$ is the magnetospheric radius, where $R_{9}$ is the WD radius in units of $10^{9} \mathrm{~cm}, L_{33}$ is the luminosity in units of $10^{33} \mathrm{erg} \mathrm{s}^{-1}$, and $\mu_{30}$ is the WD magnetic moment in units of $10^{30} \mathrm{G} \mathrm{cm}^{3}$. From the derived accretion luminosity and WD mass, we estimate a magnetic moment $\mu \sim 1.2 \times$ $10^{32} d_{1 \mathrm{kpc}} \mathrm{G} \mathrm{cm} \mathrm{cm}^{3}$. Hence, at a distance of $1 \mathrm{kpc}, \mathrm{RX} \mathrm{J} 1730$ would host a weak magnetic field WD, consistent with the fast rotation 
and pulsation properties of this source (see also below). Here we remind that also Norton et al. (2004) arrive to this conclusion for the rapid rotators in IPs.

\subsection{The white dwarf rotation}

We detected for the first time rapid X-ray pulses at the previously identified optical $128 \mathrm{~s}$ period, thus confirming this system as one of the most rapidly rotating WD IP. The X-ray pulses are highly structured and characterised by a broad and deep principal minimum and a much weaker secondary one. The principal minimum is broadly in phase with that observed in the optical modulation on the same day of the X-ray observation. There is a clear change in amplitudes and shapes moving from high energies (X-rays) to the optical (red) bands, while simultaneous observations in the far-UV range do not reveal a detectable modulation. The increase in amplitude of the X-ray pulsation with decreasing energy is a typical characteristics of IPs and consistent with photoelectric absorption in the accretion flow. The spin pulse changes from single-peaked above $5 \mathrm{keV}$ to a doublepeaked rather structured curve below $5 \mathrm{keV}$. Phasing and amplitudes suggest that accretion occurs onto two opposite polar regions (offset by $180^{\circ}$ ), with one pole (the primary) dominating in the hard X-rays. The other pole (the secondary) is essentially soft being $\sim 1.5$ times less strong than the primary pole. The highly structured shape of the spin modulation in the softer bands also suggests the presence of absorption effects especially at those phases when the secondary pole contributes most (i.e. $\phi_{\text {rot }} \sim 0.2$ ).

The strong X-ray and optical spin pulses at the $128 \mathrm{~s}$ period, indicates that accretion occurs predominantly via a disc that feeds both poles. Although we observe strong changes in the amplitude of the optical pulse from night-to-night, which is not uncommon in IPs, the stability of pulses and the absence of a beat variability at a period $P_{\omega-\Omega}=128.3 \mathrm{~s}$ in our unpublished long multi-epoch photometric optical monitoring supports the accretion disc configuration.

In the accretion curtain geometry (Rosen et al. 1988) the material flows from the accretion disc towards the magnetic poles of the WD in an arc-shaped curtain, where the optical depth is larger along the field lines. This gives rise to X-ray and optical maxima that are in phase when the curtain points away from the observer, while a minimum is observed when the polar region point towards the observer. The double-peaked shape and the phasing of X-ray and optical light curves suggest that this is the case, provided that the geometry allows the two opposite poles to come into view to the observer with different contributions. This in turn implies that the binary inclination, $i$, and magnetic colatitude, $m$, assume specific ranges of values.

Constraints on the binary inclination come from the lack of eclipses, $\cos (i)>\left(R_{\mathrm{WD}}+R_{\mathrm{sec}}\right) /$ a where a is the binary separation $\left(\sim 3.6 R_{\odot}\right.$ from the mass estimates and orbital period), giving $i \leq 70^{\circ}$. The presence of ellipsoidal variations in the optical light (Gänsicke et al. 2005) is also consistent with this angle. As discussed above, $i>50^{\circ}$, thus limiting $50^{\circ}<i<70^{\circ}$. Furthermore, the visibility of the two poles implies $|i-m|<90^{\circ}-\beta$, where $\beta$ is the subtending angle of the polar cap, which is related to the fractional accretion area by $\cos \beta=1-2 f$ (King \& Shaviv 1984). Since $f \ll 1, \beta \sim 2 f^{0.5}$ and it can be neglected at first approximation. Because the optical pulse shows similar maxima, the two poles should have similar visibility, implying that the magnetic colatitude is moderately large (see also Evans \& Hellier 2007). Absorption effects are observed in this system, suggesting $m \leq i$.

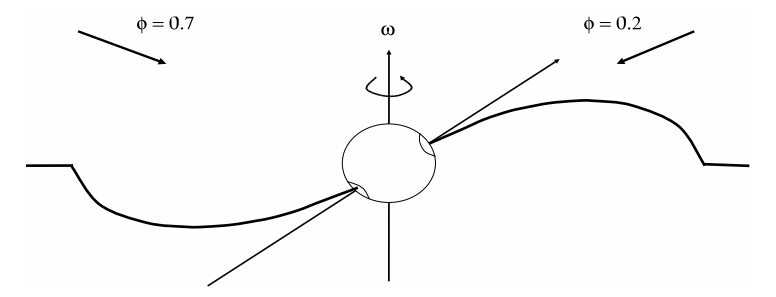

Fig. 7. A schematic picture of the accretion geometry in RXJ1730. A binary inclination $i=65^{\circ}$ and a magnetic colatitude $m=45^{\circ}$ are adopted. The phases at which the two maxima of the spin pulses occur are also denoted.

A schematic picture of the accretion flow is shown in Fig. 7 for an inclination angle $i=65^{\circ}$ and magnetic colatitude $m=45^{\circ}$. In this configuration the lower pole is better represented and contributes most at rotational phase $\sim 0.7$ when the visibility of the lower curtain is maximum. This pole (primary) is responsible for the maximum spin pulse at this phase at all energies (from hard X-rays to the optical). On the other hand, the upper pole is less represented (secondary pole) and, at rotational phase $\sim 0.2$, it is affected by absorption as indeed detected. At this phase the heated polar region of the WD atmosphere is at its maximum visibility, hence contributing most in the soft X-rays as observed. The magnetospheric radius extends up to $\sim 6 R_{\mathrm{WD}}$ suggesting that field lines reconnect far from the WD. If the magnetic colatitude is moderately large, the curtain tends to flatten in the orbital plane, allowing the outer regions of the upper curtain to be also visible and hence to contribute to the spin pulse at rotational phase 0.2 . The inner regions of the upper curtain would instead contribute little at this phase. The asymmetry in the optical pulse at spin phase $\sim 0.35$, best seen at red wavelengths, could be due to the twisting of field lines due to the fast WD rotation, which allows the upper curtain to also contribute at other phases.

The spin pulse would then be due to the combination of different contributions with likely different spectral properties and difficult to isolate. This is known to be the case in those IPs that were studied in a wide wavelength range (e.g. Welsh \& Martell 1996; de Martino et al. 1999; Eisenbart et al. 2002). The pulses in these systems were found to be multi-component, ranging from the hot WD pole or inner regions of the curtain to the cooler outer regions of the curtain itself.

In RX J1730, the pulse appears to peak in the $g^{\prime}$ band. To obtain information on the overall spectral shape of the pulse, we used the $U V W 1$ flux, the approximate average magnitudes in $u^{\prime}$, $g^{\prime}$, and $r^{\prime}$ bands (using colour relations by Fukugita et al. 1996) and the derived spin amplitudes. Using Fukugita et al. Sloan zero points and $E_{B-V}=0.45$, we obtained dereddened modulated fluxes of $F_{U V W 1} \sim 1.1 \times 10^{-15} \mathrm{erg} \mathrm{cm}^{-2} \mathrm{~s}^{-1}, F_{u^{\prime}} \sim 1.7 \times$ $10^{-15} \mathrm{erg} \mathrm{cm}^{-2} \mathrm{~s}^{-1}, F_{g^{\prime}} \sim 1.5 \times 10^{-15} \mathrm{erg} \mathrm{cm}^{-2} \mathrm{~s}^{-1}$ and $F_{r^{\prime}} \sim$ $3.7 \times 10^{-16} \mathrm{erg} \mathrm{cm}^{-2} \mathrm{~s}^{-1}$. These indicate a blackbody colour temperature of 6800-7800 K, except for the $r^{\prime}$ band whose flux is too low for any blackbody shape. Since we do not have standard star optical photometry, the above fluxes should be regarded as rough estimates. Furthermore, the extrapolation of the unabsorbed soft X-ray blackbody component to the UVW1 band predicts a flux that is 4 orders of magnitude lower than the dereddened observed one. Hence, the spin pulse appears to be dominated by relatively cool region(s) of the curtain. Although we cannot exclude the presence of the irradiated WD poles in the UV, these would be masked by the large curtain contribution. Here we remark that also the soft IP PQ Gem shows an extremely weak UV spin modulation (Evans et al. 2006), while 
others like UU Col has a strong UV spin pulsation dominated by the heated WD polar regions (de Martino et al. 2006b).

Evans \& Hellier (2007) discussed the different behaviour of IPs in terms of dipole inclinations: those soft X-ray systems, like UU Col and V405 Aur, showing double humped light curves are also found to be less affected by absorption because of highly inclined magnetic axis. RX J1730 could be an intermediate case where the magnetic dipole is moderately inclined. Furthermore, by analogy with these soft X-ray IPs that show two poles, and the polars, in which the secondary accreting pole is softer than the main pole, we might argue that RX J1730 has a similar property. Interestingly, the secondary pole in the polars is found to possess a stronger field than the primary pole (Beuermann 1999).

\subsection{The accretor in $R X J 1730$}

Fast rotational periods of the accreting WD and double-peaked $\mathrm{X}$-ray pulses were claimed to be a common feature of relatively weak magnetic field accretors (Norton et al. 1999, 2004). The very rapid WD rotation, the complex X-ray pulse and the low magnetic moment of RX J1730 (even at a distance larger than $1 \mathrm{kpc}$ ), might then add this system to the group of low magnetic field systems. Either wide or tall accretion regions could produce a double-peaked pulse profile (Norton et al. 1999). This is difficult to establish in general and in particular in RX J1730, because no spin phase-resolved X-ray spectral analysis can be performed. However, the presence of a warm absorber might favour wide accretion spots as proposed by Mukai et al. (2001). The appearance of double-humped spin light curves and soft X-ray component can be also produced by highly inclined dipole fields (Evans \& Hellier 2007). To explain the observed properties of RX J1730, the WD magnetic field axis is likely to be moderately inclined and, if indeed the WD is weakly magnetised, this system will not be prone to synchronism (Norton et al. 2004).

Furthermore, the WD is rapidly rotating but less rapidly than AE Aqr and it is neither spinning-down as instead AE Aqr does, nor spinning-up. It could be possible that RX J1730 is a young system born with a rapidly rotating WD. Very rapid rotations could be the result of a thermal timescale mass transfer phase as proposed by Schenker et al. (2002) for AE Aqr itself and other CVs that show peculiar spectral features of CNO processing. These systems could be descendant from supersoft X-ray binaries that had formerly massive secondary stars and run mass transfer on a thermal timescale that allowed nuclear stable burning onto the WD. At the end of the brief supersoft phase the systems evolve off to longer orbital period or switch to stable mass transfer. They also predict that about one-third of CVs have undergone such evolution. Signatures of non-standard evolution are spectral features due to $\mathrm{CNO}$ processing due to the chemical evolution of the donor stellar interior. These are usually observed in the UV range through anomalous line intensities of Carbon, Nitrogen and Oxygen (see also Gänsicke et al. 2003). Although we lack of UV spectroscopy to corroborate this possibility, this appears to be an interesting scenario that deserves further investigation. Also, a precise characterisation of the secondary star with adequate optical and nIR observations is needed to further progress in the understanding of this system.

RX J1730 was also recently claimed to be detected at radio wavelengths with a very likely non-thermal emission and was proposed to be a LMXB (Pandey et al. 2006). A neutron star (NS) nature and hence a LMXB was also proposed by Masetti et al. (2004) from the high luminosity that would result from locating this source in the galactic bulge. However we remark that the radio VLA survey position even including the NVSS catalogue errorbars (Condon et al. 1998), while it is within the INTEGRAL error circle, does not agree with the optical position (Gänsicke et al. 2005) and it is outside the ROSAT error circle. Hence, the claim of the radio detection at $1.4 \mathrm{GHz}$ should be taken with great caution and further observations are essential to assess this issue. We also note that very recent radio surveys (Mason \& Gray 2007) are bringing new detection of CVs in this range. It is argued that the condition to produce radio emission is that secondaries have magnetic fields of a few hundred to a few thousand Gauss (Mason \& Gray 2007; Meintjes \& Jurua 2006). It is further suggested that the systems should be disc-less because the few $\mathrm{CV}$ s identified in the radio regime are polars and pre-CVs. The only IP with a secure radio counterpart again is the peculiar system AE Aqr. Though the fast rotation and the long orbit of RX J1730 might bring similarities to that system, AE Aqr is a strongly flaring source at all wavelengths and it is quite atypical in its X-ray emission, being a soft X-ray source with a very low accretion rate (see Itoh et al. 2006, and references therein). From the detection of a spin-down of the WD, it is now well established that this system is in a propeller state (Schenker et al. 2002, and references therein.) RX J1730 is not observed to be a flaring source and its spin period is stable at the quoted value. The analysis of the XMM-Newton and INTEGRAL data reveals many similarities with other IPs. It is a very hard X-ray source, though, in some respect, peculiar in its soft emission. The timing analysis shows that RX J1730 is modulated at the WD spin period up to $10 \mathrm{keV}$, implying that the primary is experiencing accretion via a disc. It is therefore difficult to match the observed characteristics of RX J1730 with either a state similar to AE Aqr or a neutron star accretor.

\section{Conclusions}

We presented the first X-ray study of RXJ1730 based on an $X M M$-Newton observation, complemented with INTEGRAL and optical fast photometric data that confirm this $\mathrm{CV}$ as a magnetic system of the IP type.

The rapid $128 \mathrm{~s}$ pulsation is energy dependent with remarkable differences from the X-rays, the UV to the optical ranges. We identified two emitting poles with different contributions. A main emitting pole contributes from soft to the hard X-rays, while the secondary pole contributes most in the soft bands. The WD magnetic dipole field should be moderately inclined, thus allowing the visibility of both poles, with the lower pole better represented. The optical spin pulse is mainly due to reprocessing in the accretion flows onto the WD and dominated by the outer regions. The lack of detectable UV pulsation is an unexpected property, which might indicate that reprocessing at both the WD surface and in the inner regions of the accretion flow is masked by the contribution of the outer flow regions.

$\mathrm{X}$-ray spectral analysis reveals a hard X-ray spectrum as well as remarkable soft X-ray complexities. These can be identified in a warm absorber, which makes this system the second IP where an absorption edge from ionised oxygen has been revealed, together with multiple optically thin emissions and a blackbody component. The last adds RXJ1730 to the small group of soft $\mathrm{X}$-ray IPs. We however find a large luminosity for this component that is difficult to explain. The observed X-ray properties are consistent with a system hosting a weakly magnetised WD rather than a NS. The long $15.4 \mathrm{~h}$ orbital period and rapid $128 \mathrm{~s}$ WD rotation could suggest that RXJ1730 is a young IP born with a rapidly rotating WD. To understand its evolutionary status, this system deserves further observations especially addressing the donor star parameters. 
Acknowledgements. D.D.M. acknowledges financial support by the Italian Space Agency (ASI) under contract I/023/05/0 and thanks R. Gonzalez-Riestra at ESAC for her help in checking operational performance of the OM during the observation. ULTRACAM is supported by PPARC grant PP/D002370/1. The WHT is operated on the island of La Palma by the Isaac Newton Group in the Spanish Observatorio del Roque de los Muchachos of the Instituto de Astrofsica de Canarias.

\section{References}

Anders, E., \& Grevesse, N. 1989, GeCoA, 53, 197

Barlow, E. J., Knigge, C., Bird, A. J., \& Dean, A. 2006, MNRAS, 372, 224

Beuermann, K. 1999, MPE Report, 272, 410

Bird, A. J., Barlow, E. J., Bassani, L., et al. 2004, ApJ, 607, 33

Bird, A. J., Malizia, A., Bazzano, A., et al. 2007, ApJS, 170, 175

Bonnet-Bidaud, J.-M., Mouchet, M., de Martino, D., \& Silvotti, R. 2006, A\&A, 445, 1037

Bonnet-Bidaud, J.-M., de Martino, D., Falanga, M., Mouchet, M., \& Masetti, N. 2007, A\&A, 473, 185

Collier Cameron, A. 2002, ASPC, 261, 11

Casares, J., Mouchet, M., Martinez-Pais, I. G., et al. 1996, MNRAS, 282, 182

Condon, J. J., Cotton, W. D., Greisen, E. W., et al. 1998, AJ, 115, 1693

Cumming, A. 2002, MNRAS, 333, 589

de Martino, D., Silvotti, R., Buckley, D. A. H., et al. 1999, A\&A, 350, 517

de Martino, D., Matt, G., Mukai, K., et al. 2001, A\&A, 377, 499

de Martino, D., Matt, G., Belloni, T., et al. 2004, A\&A, 415, 1009

de Martino, D., Matt, G., Mukai, K., et al. 2005, A\&A, 437, 935

de Martino, D., Matt, G., Mukai, K., et al. 2006a, ESA SP-604, 261

de Martino, D., Matt, G., Mukai, K., et al. 2006b, A\&A, 454, 287

de Martino, D., Bonnet-Bidaud, J.-M., Mouchet, M., et al. 2006c, A\&A, 449, 1151

den Herder, J. W., Brinkman, A. C., Kahn, S. M., et al. 2001, A\&A, 365, L7 Dhillon, V., Marsh, T. R., Stevenson, M. J., et al. 2007, MNRAS, 378, 825

Done, C., Mulchaey, J. S., Mushotzky, R. F., \& Arnaud, K. A. 1992, ApJ, 395, 275

Eisenbart, S., Beuermann, K., Reinsch, K., \& Gänsicke, B. T. 2002, A\&A, 382, 984

Evans, P. A., \& Hellier, C. 2004, MNRAS, 353, 447

Evans, P. A., \& Hellier, C. 2007, ApJ, 663, 1277

Evans, P. A., Hellier, C., \& Ramsay, G. 2006, MNRAS, 369, 1229 Fukugita, M., Ichikawa, T., Gunn, J. E., et al. 1996, AJ, 111, 1748

Falanga, M., Bonnet-Bidaud, J.-M., \& Suleimanov, V. 2005, A\&A, 444, 561

Gänsicke, B. T., Szkody, P., de Martino, D., et al. 2003, ApJ, 594, 443

Gänsicke, B. T., Marsh, T. R., Edge, A., et al. 2005, MNRAS, 361, 141

Grevesse, N., Asplund, M., \& Sauval, A. J. 2007, SSRv, in press

Haberl, F., \& Motch, C. 1995, A\&A, 297, L37
Haberl, F., Motch, C., \& Zickgraf, F.-J. 2002, A\&A, 387, 201

Itoh, K., Okada, S., Ishida, M., \& Kunieda, H. 2006, ApJ, 639, 397

James, C. H., Ramsay, G., Cropper, M., \& Branduardi-Raymont, G. 2002, MNRAS, 336, 550

Jansen, F., Lumb, D., Altieri, B., et al. 2001, A\&A, 365, L1

King, A. R., \& Shaviv, G. 1984, MNRAS, 211, 883

Lebrun, F., Leray, J.-P., Lavocate, Ph., et al. 2003, A\&A, 411, L141

Magdziarz, P., \& Zdziarski, A. A. 1995, MNRAS, 273, 837

Masetti, N., Palazzi, E., Bassani, L., et al. 2004, A\&A, 426, L41

Masetti, N., Bassani, L., Bazzano, A., et al. 2006a, A\&A, 455, 11

Masetti, N., Pretorius, M. L., Palazzi, E., et al. 2006b, A\&A, 449, 1139

Mason, P. A., \& Gray, C. L. 2007, ApJ, 660, 662

Mason, K. O., Breeveld, A., Much, R., et al. 2001, A\&A, 365, L36

Matt, G., Perola, G. C., \& Piro, L. 1991, A\&A, 247, 25

Matt, G., de Martino, D., Gänsicke, B. T., et al. 2000, A\&A, 358, 177

McDermott, P. N., \& Taam, R. E. 1989, ApJ, 342, 1019

Meintjes, P. J., \& Jurua, E. 2006, MNRAS, 372, 1279

Morales-Rueda, L., Still, M. D., Roche, P., et al. 2002, MNRAS, 329, 597

Mukai, K., Ishida, M., \& Osborne, J. P. 1994, PASJ, 46, L87

Mukai, K., Ishida, M., \& Osborne, J. P. 1995, ASPC, 85, 119

Mukai, K., Kallman, T., Schlegel, E., et al. 2001, ASPC, 251, 90

Norton, A. J., Beardmore, A. P., Allan, A., \& Hellier, C. 1999, A\&A, 347, 293

Norton, A. J., Wynn, G. A., \& Somerscales, R. V. 2004, ApJ, 614, 349

Pandey, M., Rao, A. P., Manchanda, R., et al. 2006, MNRAS, 453, 83

Patterson, J. 1994, PASP, 106, 209

Piconcelli, E., Jimenez-Bailon, E., Guainazzi, M., et al. 2004, MNRAS, 351, 161

Piconcelli, E., Jimenez-Bailon, E., Guainazzi, M., et al. 2005, A\&A, 432, 15

Ramsay, G., \& Cropper, M. 2004, MNRAS, 347, 497

Ramsay, G., Cropper, M., Hellier, C., \& Wu, K. 1998, MNRAS, 297, 1269

Ritter, H., \& Kolb, U. 2003, A\&A, 404, 301 (update RKcat7.6 in 2006)

Rodriguez-Gil, P., Gänsicke, B. T., Hagen, H.-J., et al. 2005, A\&A, 440, 701

Rosen, S. R., Mason, K. O., \& Cordova, F. A. 1988, MNRAS, 231, 549

Schenker, K., King, A. R., Kolb, U., et al. 2002, MNRAS, 337, 1105

Southworth, J., Gänsicke, B. T., Marsh, T. R., et al. 2006, MNRAS, 373, 687

Southworth, J., Gänsicke, B. T., Marsh, T. R., et al. 2007, MNRAS, 378, 635

Strüder, L., Briel, U., Dennerl, K., et al. 2001, A\&A, 365, L18

Taam, R., \& Spruit, H. 2002, ApJ, 561, 329

Turner, M. J. L., Abbey, A., Arnaud, M., et al. 2001, A\&A, 365, L27

Ubertini, P., Lebrun, F., Di Cocco, G., et al. 2003, A\&A, 411, L131

Venter, L. A., \& Meintjes, P. J. 2007, MNRAS, 378, 681

Verner, D. A., Ferland, G. J., Korista, K. T., \& Yakovlev, D. G. 1996, ApJ, 465, 487

Warner, B. 1995, Cataclysmic Variables (Cambridge: Cambridge Univ. Press)

Warner, B., \& Woudt, P. 2004, ASPC, 315, 39

Welsh, W. F., \& Martell, P. J. 1996, MNRAS, 282, 739

Wheatley, P. J., Marsh, T. R., \& Clarkson, W. 2006, ATel, 765

Wilms, J., Allen, A., \& McCray, R. 2000, ApJ, 542, 914

Winkler, C., Courvoisier, T. J.-L., Di Cocco, G., et al. 2003, A\&A, 411, L1 